\title{
Couplage et adaptation de maillage anisotrope pour des simulations de flux d'air dans des géométries complexes
}

\author{
Cécile Dobrzynski - Pascal Frey — Olivier Pironneau \\ Université Pierre et Marie Curie - Laboratoire J-L Lions \\ 175 rue du Chevaleret F-75013 Paris \\ dobrzyns@ann.jussieu.fr
}

\begin{abstract}
RÉSUMÉ. La simulation des écoulements d'air au sein des bâtiments est un enjeu d'importance pour de nombreuses industries. Les objectifs diffèrent d'une application à l'autre, allant de l'optimisation des systèmes d'air conditionné dans des bâtiments à l'étude de la dispersion de produits nocifs via le système de ventilation. Dans ce papier, le problème que nous traitons concerne le couplage entre les équations de Navier-Stokes pour les fluides incompressibles et une équation de température avec flux convectif. Ce problème est résolu en utilisant une stratégie d'adaptation de maillage. Les maillages sont générés et adaptés via une méthode de remaillage local basée sur une version anisotrope du noyau de Delaunay.

ABSTRACT. The numerical simulation of air flow in a building or a house is certainly a very challenging field of engineering applications. The objectives differ from one application to the other, ranging from the optimization of air conditioning systems in buildings to the study of the propagation of harmful products via the air conditioning system. In this paper, we address the problem of coupling the Navier-Stokes equations for incompressible fluids and a temperature equation. To solve this problem in $3 d$, we use an anisotropic mesh adaptation strategy. The meshes are generated by a local remeshing techniques based on an anisotropic version of Delaunay kernel. The method is fairly general and applies to other cases as well. We illustrate this problem with an example showing the heating of the last floor of a furnished house.

MOTS-CLÉS : adaptation de maillages ; Navier-Stokes incompressible ; CFD ; maillages anisotropes $3 d$; méthodes de remaillage local.

KEYWORDS: mesh adaptation; Navier-Stokes incompressible; CFD; $3 d$ anisotropic meshes; local remeshing techniques.
\end{abstract}

DOI:10.3166/REMN.16.749-773 @ 2007 Lavoisier, Paris

REMN - 16/2007. Modélisations numériques en mécanique, pages 749 à 773 


\section{Introduction}

On s'intéresse dans cet article aux problèmes d'ingénierie traitant d'air et de température. Le spectre de ces problèmes est très large et la finalité de certaines études est le dimensionnement et le positionnement des bouches d'air conditionné dans des bâtiments. Ce genre de problèmes relève du domaine de l'architecture, cependant, rares sont les études utilisant des modèles précis pour simuler les écoulements d'air dans les bâtiments. Les méthodes utilisées considèrent les échanges de température à l'aide d'une formule empirique qui fait intervenir la taille des fenêtres, la taille des pièces, la nature des matériaux, etc. Lorsque le but est de connaître la température en tout point d'un domaine ainsi que la vitesse du flux en ces points (pour évaluer par exemple la force d'un courant d'air), des simulations de mécanique des fluides sont alors indispensables.

Du point de vue de la modélisation, il est bien établi que, pour une température comprise entre -20 et 100 degrés, l'air ambiant est un fluide newtonien compressible dont la température et la densité sont reliées par la loi des gaz parfaits. Il nous faut donc résoudre les équations de Navier-Stokes pour les fluides incompressibles corrigées avec un terme de Boussinesq, puis les coupler avec une équation de température (Landau et al., 1964). Ce problème est non linéaire pour les variables couplées de vitesse, de pression et de température. Nous adopterons un algorithme maintenant classique (Chorin, 1967, Rannacher, 1992) qui consiste à linéariser et à découpler le problème à chaque pas de temps. Un couplage faible sera donc mis en place : on calcule tout d'abord la vitesse, puis la pression, et enfin la température.

Ce genre de simulations nécessite en principe le recours à des modèles de turbulence, en particulier le modèle $k-\epsilon$ avec des lois de parois (Mohammadi et al., 1994), que nous n'évoquerons pas ici. En effet, la construction de couches limites dans le maillage du domaine est indispensable pour mener à bien de telles simulations. Mais celle-ci est délicate dans des maillages tridimensionnels et nos mailleurs ne permettent pas encore de la générer de manière automatique.

La résolution des équations de Navier-Stokes incompressibles par des méthodes d'éléments finis est fiable et efficace (Glowinski, 1984, Girault et al., 1986, Pironneau, 1989). La principale difficulté de la mise en œuvre de telles simulations réside dans le temps nécessaire pour les calculs. Dans le but d'accélérer les temps de calcul et d'effectuer des calculs précis, nous avons exploré deux pistes : d'une part, la parallélisation des codes de calcul et d'autre part, la mise en place de méthodes d'adaptation de maillages.

L'approche que nous avons choisie pour l'adaptation de maillage est une hméthode basée sur un estimateur de l'erreur d'interpolation permettant la construction d'une carte de métrique anisotrope (Vallet, 1992, Alauzet, 2003). Du point de vue de la technologie de maillage, nous avons choisi de construire un maillage anisotrope par une méthode de remaillage local qui, de par sa nature, est particulièrement bien adaptée à ce genre de problème. On peut mettre en avant plusieurs intérêts à ces méthodes : 
i) à tout moment de la procédure de remaillage, le maillage est valide et conforme (au sens des éléments finis),

ii) le nombre d'opérations diminue au fil des itérations (minimisant donc les erreurs d'interpolation) pour un phénomène stationnaire.

Dans cette optique, nous avons mis en œuvre une méthode de remaillage local d'un maillage tétraédrique basée sur une carte de métrique anisotrope et dans laquelle l'insertion des points se fait par une extension anisotrope du noyau de Delaunay.

Dans ce papier, nous commençons par rappeler la méthode d'adaptation de maillage choisie. La deuxième section est consacrée à la construction d'un maillage anisotrope par une méthode de remaillage local. Nous expliquons ensuite la résolution du problème de mécanique des fluides traité puis nous l'illustrons sur l'exemple du réchauffement d'une maison de trois étages.

\section{Méthode d'adaptation de maillage stationnaire}

\subsection{Estimateur d'erreur}

Dans un contexte de simulations numériques, l'adaptation de maillages est une méthode qui vise à contrôler l'erreur d'approximation faite sur un maillage. La première étape de cette méthode consiste donc à trouver un estimateur de cette erreur. Le lemme de Céa, nous dit que pour les équations elliptiques, l'erreur d'approximation est majorée par l'erreur d'interpolation (Ciarlet, 1991). Des études expérimentales ont montré qu'on pouvait généraliser ceci à d'autres types d'équations. On considère donc que l'erreur d'approximation est majorée par l'erreur d'interpolation (Fortin, 2000) et on cherche à majorer celle-ci.

Théorème 1 Pour un maillage composé de simplexes $K$, on a la majoration suivante de l'erreur d'interpolation sur un élément (Alauzet et al., 2003) :

$$
\left\|u-\Pi_{h} u\right\|_{\infty, K} \leq c \max _{x \in K} \max _{\vec{v} \subset K}\left\langle\vec{v},\left|H_{u}(x)\right| \vec{v}\right\rangle
$$

avec $u$ une fonction de $\mathbb{R}^{3}$ dans $\mathbb{R}, \Pi_{h}$ u l'interpolé linéaire de u sur $K, H_{u}(x)$ le hessien de u et c une constante.

La majoration donnée par le théorème précédent n'est pas exploitable telle quelle puisqu'elle fait intervenir deux maxima qui ne peuvent pas être évalués numériquement à un coût raisonnable. Pour supprimer le maximum associé aux vecteurs inclus dans $K$, on fait intervenir les arêtes du maillage dans la majoration de l'erreur : en effet, on peut montrer que tout vecteur $\vec{v} \subset K$ peut s'écrire comme une combinaison linéaire des arêtes de $K$. La majoration de l'erreur d'interpolation devient donc :

$$
\left\|u-\Pi_{h} u\right\|_{\infty, K} \leq c \max _{x \in K} \max _{\vec{e} \in E_{K}}\left\langle\vec{e},\left|H_{u}(x)\right| \vec{e}\right\rangle .
$$


En pratique, le maximum sur le champ de métrique $\left|H_{u}(x)\right|$ n'est pas connu, ce qui rend délicat l'évaluation de l'estimateur. Pour y remédier, on va exhiber sur $K$ un tenseur de métrique $\mathcal{M}(K)$ vérifiant :

$$
\max _{x \in K}\left\langle\vec{e},\left|H_{u}(x)\right| \vec{e}\right\rangle \leq\langle\vec{e}, \mathcal{M}(K) \vec{e}\rangle, \text { pour tout } \vec{e} \in E_{K}
$$

et tel que la région définie par: $\{\langle\vec{v}, \mathcal{M}(K) \vec{v}\rangle \mid \forall \vec{v} \subset K\}$ soit de volume minimal. On obtient donc la majoration explicite suivante :

$$
\left\|u-\Pi_{h} u\right\|_{\infty, K} \leq c \max _{\vec{e} \in E_{K}}\langle\vec{e},|\mathcal{M}(K)| \vec{e}\rangle
$$

où $c$ est une constante (en théorie dépendante de la dimension de l'espace).

\subsection{Schéma d'adaptation stationnaire}

Le schéma d'adaptation de maillage est d'application générale, c'est-à-dire indépendant du domaine d'application. Il peut être décomposé en cinq étapes :

0) soit $\mathcal{H}_{i}$ le maillage de l'itération $i$ et $u_{i}$ une solution associée aux nœuds de $\mathcal{H}_{i}$,

1) calculer la solution $u_{i+1}$ sur le maillage $\mathcal{H}_{i}$,

2) calculer un tenseur de métrique discrète $\mathcal{M}_{i}$ à partir de l'estimateur d'erreur géométrique,

3) adapter le maillage $\mathcal{H}_{i}$ en utilisant $\mathcal{M}_{i}$,

4) interpoler la solution $u_{i}$ sur le nouveau maillage $\mathcal{H}_{i+1}$ et reprendre du début avec $i=i+1$.

Nous allons détailler les points 2 et 3 dans les sections suivantes. Le point 4 relatif à l'interpolation est expliqué dans (Alauzet et al., 2003).

\subsection{Adaptation de maillage}

L'adaptation de maillage a pour but d'équirépartir l'erreur d'approximation sur un maillage. Pour cela, on fixe une valeur admissible $\varepsilon$ pour l'erreur d'interpolation et on veut caractériser les éléments $K$ du maillage sous cette contrainte. D'après la majoration 1 , la relation suivante doit donc être vérifiée :

$$
\forall \vec{e} \in E_{K},\langle\vec{e}, \tilde{\mathcal{M}}(K) \vec{e}\rangle=1 \Longleftrightarrow \forall \vec{e} \in E_{K}, \quad\left(l_{\tilde{\mathcal{M}}(K)}(\vec{e})\right)^{2}=1 .
$$

avec $E_{K}$ l'ensemble des arêtes du maillage et $\tilde{\mathcal{M}}(K)=\frac{c}{\varepsilon} \mathcal{M}(K)$.

Cette relation signifie que l'erreur d'interpolation sur un élément $K$ est de l'ordre de $\varepsilon$ si la longueur des arêtes de $K$ vaut 1 dans la métrique $\tilde{\mathcal{M}}(K)$ c'est-à-dire si les arêtes sont de longueur unité. 
Pour construire un maillage adapté, on est donc conduit à modifier la notion de produit scalaire qui sous-tend la notion de longueur utilisée par les générateurs de maillages. On est amené à construire un maillage avec des arêtes unité dans la métrique $\tilde{\mathcal{M}}$. Cette manière de traiter la construction d'un maillage adapté est intéressante : la généreration des maillages peut être basée sur l'étude des longueurs d'arêtes et non sur celle de la taille des éléments. En effet, dans une méthode de génération de maillages gouvernée par une carte de métrique, on peut assurer la conformité des éléments avec cette carte par un contrôle sur les longueurs d'arêtes.

Pour pouvoir appliquer tout ceci à un calcul de mécanique des fluides, il nous reste à préciser comment évaluer le tenseur de métrique $\tilde{\mathcal{M}}$.

\subsection{Création de la métrique}

Un champ de métrique sur un domaine $\Omega$ est une donnée de dimension infinie qui n'est pas exploitable numériquement. Une approximation discrète de la métrique qui utilise comme support le maillage sera donc utilisée. Cette métrique sera définie aux sommets du maillage. Par la suite ce champ de métrique discret sera rendu continu sur tout le domaine en utilisant un schéma d'interpolation (Alauzet et al., 2003, Leservoisier et al., 2001).

En pratique, on veut équirépartir une erreur d'interpolation $\varepsilon$ fixée sur le maillage. Un tenseur de métrique en dimension trois est construit de la manière suivante :

$$
\tilde{\mathcal{M}}=\mathcal{R} \tilde{\Lambda} \mathcal{R}^{-1} \text {, où } \tilde{\Lambda}=\left(\begin{array}{ccc}
\tilde{\lambda}_{1} & 0 & 0 \\
0 & \tilde{\lambda}_{2} & 0 \\
0 & 0 & \tilde{\lambda}_{3}
\end{array}\right)
$$

dans lequel,

$$
\tilde{\lambda}_{i}=\min \left(\max \left(c \varepsilon^{-1}\left|\lambda_{i}\right|, h_{\max }^{-2}\right), h_{\min }^{-2}\right),
$$

avec $\mathcal{R}$ la matrice des vecteurs propres, les $\left(\lambda_{i}\right)_{i=1 . .3}$ les valeurs propres de la matrice hessienne $H_{u}$, c la constante qui apparaît dans la majoration de l'erreur d'interpolation, $h_{\min }$ (resp. $h_{\max }$ ) la taille minimale (resp. maximale) autorisée pour les arêtes du maillage. Ces dernières sont introduites pour éviter les métriques « irréalistes » (nulles ou infinies). Le tenseur de métrique ainsi défini est de nature anisotrope : les directions principales sont données par les vecteurs propres du hessien et les tailles relatives respectives par les $\tilde{\lambda}_{i}$.

Le calcul de la métrique étant lié à la matrice hessienne des variables du problème, il est donc important d'en avoir une bonne évaluation numérique si on veut une métrique précise. Cependant en pratique, la solution exacte du problème étudié n'étant pas connue, il faut utiliser la solution discrète du problème qui, dans notre cas, est $P^{1}$ pour évaluer le hessien. En d'autres termes, le hessien de la solution approchée est nul 
à l'intérieur des éléments et une masse de Dirac sur leur frontière. Il faut donc reconstruire une approximation du hessien de la solution à partir de la solution discrète par des méthodes numériques (double projection locale $L^{2}$, méthode basée sur la formule de Green, reconstruction par moindres carrés...) (Alauzet, 2003).

\section{Construction d'un maillage anisotrope par des méthodes de remaillage local}

\subsection{Motivation}

Il existe deux types d'approche pour construire un maillage adapté : une approche dite globale et une autre dite locale. L'approche globale consiste à reconstruire intégralement un maillage dont les tailles (et les directions) des éléments seront en accord avec la métrique spécifiée. L'approche locale quant à elle modifie itérativement un maillage existant (Frey et al., 1999).

Plusieurs travaux préconisent l'approche locale pour construire un maillage tétraédrique adapté (Coupez, 2000, Tam et al., 2000, Pain et al., 2001, Remacle et al., 2002). Cette méthode nous paraît une approche intéréssante. Tout d'abord, elle préserve la validité et la conformité : le maillage étant modifié pas à pas, la procédure peut être arrêtée à tout moment. De plus, comme les problèmes auxquels on s'intéresse, tendent vers une solution stationnaire, au fil des itérations de la boucle d'adaptation, le maillage devrait être de moins en moins modifié. Ceci entraînera d'une part, la diminution du temps de maillage et d'autre part, l'absence d'erreur d'interpolation sur la solution sur les points qui resteront inchangés.

Notre approche est basée sur l'étude de la longueur des arêtes : les arêtes trop longues seront subdivisées en arêtes de longueur unité et à l'inverse, celles trop courtes seront supprimées. Les méthodes de Delaunay nous paraissent un choix judicieux pour insérer des points dans le maillage. En effet, ce genre de technique permet de connecter les points de manière à prendre en compte des directions prescrites par une métrique. Par conséquent, l'insertion des points dans le maillage se fera via une version anisotrope $3 \mathrm{~d}$ d'une méthode incrémentale basée sur le noyau de Delaunay.

\subsection{Définitions}

Soient $P$ un point et $e$ une arête d'un maillage $\mathcal{T}$.

Définition 1 La cavité $\mathcal{C}_{P}$ du point $P$ est constituée de tous les éléments de $\mathcal{T}$ dont la boule circonscrite contient le point $P$ :

$$
\mathcal{C}_{P}=\{K \in \mathcal{T} \mid P \in D(K)\} .
$$

Définition 2 La boule $\mathcal{B}_{P}$ du point $P$ est l'ensemble des éléments de $\mathcal{T}$ contenant le point $P$ :

$$
\mathcal{B}_{P}=\{K \in \mathcal{T} \mid P \in K\} .
$$


Définition 3 La coquille $\mathcal{S}_{e}$ de l'arête e est composée de tous les éléments de $\mathcal{T}$ contenant cette arête :

$$
\mathcal{S}_{e}=\{K \in \mathcal{T} \mid e \in K\}
$$

Pour juger rapidement la qualité d'un maillage par rapport à une carte donnée, on définit l'indice d'efficacité d'un maillage. On note $l_{i}$ la longueur de l'arête $e_{i}$ par rapport à une métrique donnée.

Définition 4 L'indice d'efficacité d'un maillage est défini comme l'exponentielle de la moyenne entre les différences à 1 de toutes les longueurs $l_{i}$ des arêtes d'un maillage normalisé par le nombre d'arêtes du maillage. Si on note $\tau$ cet indice, on a:

$$
\tau=\exp \left(\frac{1}{n e} \sum_{i=1}^{n e} e_{i}\right)
$$

avec $e_{i}=l_{i}-1$ si $l_{i}<1$ et $e_{i}=\frac{1}{l_{i}}-1$ si $l_{i}>1$ et ne le nombre d'arêtes du maillage.

Définition 5 On définit la mesure de qualité d'un élément $K$ suivante :

$$
Q_{K}=\leq_{\max } \frac{\sum_{1 \leq i<j \leq 4} S_{j}}{\sqrt{\operatorname{Det}\left(\mathcal{M}_{K}\right)} V_{K}}
$$

avec $S_{j}$ la surface de la face $j$ dans la métrique $\mathcal{M}_{K}$, $l_{\text {max }}$ la plus grande arête de $K$ dans $\mathcal{M}_{K}$ et $V_{K}$ le volume de $K$.

Un tel critère de qualité permet de décider si un simplexe est acceptable ou non. Notons que d'après la formule ci-avant, la qualité $Q_{K}$ calculée varie dans $[1,+\infty$ [ et que plus le volume du tétraèdre est petit et plus $Q_{K}$ sera grand. On évalue donc la dégradation des tétraèdres.

\subsection{Calcul de longueurs}

\subsubsection{Longueur unité}

Pour construire un maillage adapté on a besoin de savoir calculer les longueurs des arêtes dans le cas où la métrique varie en tout point de l'espace. En effet, chaque matrice $\mathcal{M}$ définit une expression particulière pour le produit scalaire $\langle,\rangle_{\mathcal{M}}$. Afin de simplifier les interactions entre métriques distinctes et au lieu de construire des arêtes de longueurs quelconques dans chaque métrique, on va par convention les construire toutes de longueur 1 . 
Définition 6 On dit que le vecteur $\vec{v}$ est de longueur unité dans la métrique $\mathcal{M}$ si et seulement si $\|\vec{v}\|_{\mathcal{M}}=1$.

Lors de la construction du nouveau maillage, on veut donc insérer les nouveaux points en créant des arêtes de longueur 1 : si $P$ est un point du maillage auquel est associée la métrique $\mathcal{M}$, on veut insérer le point $X$ tel que l'arête $P X$ ait une longueur égale (ou proche) de 1 dans la métrique de $\mathcal{M}(P)$, c'est-à-dire :

$$
l_{\mathcal{M}(P)}(P X)=\langle\overrightarrow{P X}, \overrightarrow{P X}\rangle_{\mathcal{M}(P)}^{\frac{1}{2}}=\sqrt{t \overrightarrow{P X} \mathcal{M}(P) \overrightarrow{P X}}=1 .
$$

\subsubsection{Longueur moyenne}

En pratique, une métrique différente est prescrite en chaque sommet du maillage. Pour calculer la longueur de l'arête $A B$, on voudrait tenir compte à la fois de la métrique donnée en $A$ et de celle donnée en $B$ ainsi que des métriques de tous les points intermédiaires du segment $A B$.

Pour cela, on définit la longueur moyenne sur toutes les métriques de la manière suivante (George et al., 2004) :

$$
l_{\mathcal{M}}(\overrightarrow{A B})=\int_{0}^{1} \sqrt{t \overrightarrow{A B} \mathcal{M}(A+t \overrightarrow{A B}) \overrightarrow{A B}} d t
$$

\subsection{Opérateurs de modifications locales}

Les outils d'optimisation de maillage peuvent être classés en deux catégories selon que les modifications soient topologiques ou géométriques. L'opérateur topologique est le retournement d'arêtes : il modifie uniquement les connexions entre les points d'un maillage. Par contre, les opérateurs géométriques (fusion de sommets et bougé de points) modifient la position ou le nombre de points du maillage.

\subsubsection{Retournements d'arêtes}

Le retournement d'arête ou bascule d'arête est une opération topologique qui agit sur les tétraèdres contenus dans la coquille de l'arête.

Notons que dans un maillage, les coquilles peuvent être ouvertes (par exemple dans le cas d'une arête de peau) ou fermées. On ne s'intéresse ici qu'au retournement d'arête ayant des coquilles fermées, autrement dit aux arêtes internes du maillage.

L'opérateur généralisé de bascule d'arête a pour but de supprimer une arête du maillage (et donc tous les tétraèdres la contenant) tout en gardant un maillage valide. Pour procéder à un retournement d'arête, on associe à une coquille un pseudo- 
polygone dont les sommets sont tous les sommets contenus dans les tétraèdres de la coquille qui ne sont pas les extrémités de l'arête traitée (figure 1).
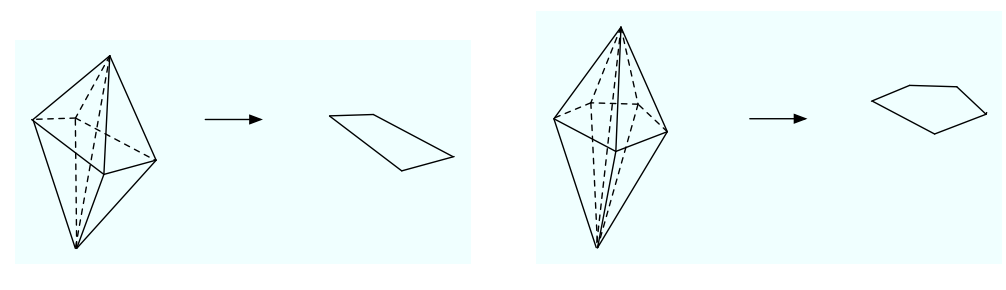

Figure 1. Coquilles d'arête et «pseudo» polygones associés

L'opérateur généralisé de bascule d'arête consiste à examiner toutes les triangulations possibles d'un polygone associé à l'arête. Une fois une triangulation du polygone choisie, il suffit de relier les triangles des polygones aux extrémités de l'arête formant ainsi des couples de tétraèdres.

Le nombre de Catalan d'ordre $n$ :

$$
\operatorname{Cat}(n)=\frac{(2 n-2) !}{n !(n-1) !},
$$

donne le nombre maximal de triangulation topologiquement possible pour une coquille contenant $n+1$ éléments.

Ce nombre permet d'évaluer la complexité d'un tel opérateur : pour une coquille contenant 8 tétraèdres, il y aura 132 possibilités à tester. Dans notre cas, nous nous sommes limités à considérer les retournements d'arêtes qui ont au plus 7 tétraèdres dans leur coquille, ceci couvrant la majorité des arêtes d'un maillage.

On utilise l'opérateur de bascule d'arête pour améliorer la qualité des éléments du maillage. Cette procédure se décompose en deux étapes. La première consiste à s'assurer que la bascule est admissible. Par une simulation de celle-ci, on teste si chaque nouveau tétraèdre $K$ est valide et s'il vérifie la condition suivante :

$$
\forall K_{i} \in \mathcal{S}_{e} \quad Q_{K}<Q_{K_{i}}
$$

Si ceci est vrai pour tous les nouveaux tétraèdres, la bascule est appliquée : l'arête est enlevée du maillage par la suppression de tous les tétraèdres contenus dans sa coquille. Les nouveaux tétraèdres sont ensuite créés.

\subsubsection{Fusion de sommets}

On s'intéresse dans cette section à l'opérateur de suppression d'une arête. Cette opération est principalement utilisée lorsqu'une arête du maillage est « trop courte ».

Etant donné une arête $A B$, il s'agit ici de la réduire à un point. Plusieurs solutions apparaissent alors : soit l'arête est réduite à un de ses sommets (ce qui revient à supprimer l'autre sommet du maillage), soit elle est réduite en un point choisi sur $A B$. En 
pratique, pour fusionner une arête, il faut s'assurer que la boule du point résultant est valide.

Dans notre cas, nous voulons fusionner une arête tout en s'assurant de ne pas créer d'arêtes trop longues. Il faut donc rajouter une vérification sur les longueurs des nouvelles arêtes pour valider la fusion.

\subsubsection{Bougé de points}

Le bougé de points maintient la connectivité du maillage. Cette procédure a pour but d'améliorer la qualité des éléments du maillage tout en prenant en compte les longueurs prescrites. Autrement dit, on cherche une nouvelle position $P^{\prime}$ pour le point $P$ telle que :

- tous les tétraèdres contenant le point $P^{\prime}$ aient une meilleure qualité que le plus mauvais des tétraèdres contenant $P$,

- toutes les arêtes issues du point $P^{\prime}$ soient de longueurs admissibles.

Il est possible de répondre à ce problème par la résolution d'une EDP mais nous avons privilégié une approche locale de relaxation de type Gauss-Seidel.

La détermination de la position optimale pour le point $P$ est basée uniquement sur les longueurs des arêtes incidentes à ce point (les directions prescrites par les métriques ne sont donc pas prises en compte). Soit $n_{b}$ le nombre de tétraèdres contenus dans la boule de $P$.

On commence par calculer, pour chaque tétraèdre $i$ contenu dans la boule du point $P$, la position optimale $P_{i}^{o p t}$ du point $P$. Notons $F_{P}$ la face du tétraèdre $i$ opposée à $P$. La position $P_{i}^{\text {opt }}$ dépend de la longueur prescrite en chaque point de la face $F_{P}$. Pour chaque point $P_{j}$ de la face $F_{P}$, on calcule une position pour le point $P$ telle que $l\left(P P_{j}\right)=1$, le point $P_{i}^{o p t}$ est ensuite défini comme le barycentre de ces positions :

$$
P_{i}^{o p t}=\frac{1}{3} \sum_{j=1}^{3}\left(P+\frac{\overrightarrow{P P_{j}}}{l\left(P P_{j}\right)}\right)
$$

Enfin, la position optimale $P^{\prime}$ est calculée par relaxation sur le barycentre de tous les $P_{i}^{\text {opt }}$ :

$$
P^{\prime}=(1-\omega) P+\omega\left(\frac{1}{n_{b}} \sum_{i=1, . ., n_{b}} P_{i}^{o p t}\right),
$$

où $\omega$ est un paramètre de relaxation compris entre 0 et 1.

On choisit $\omega$ le plus grand possible tel que :

1) en moyenne les longueurs des arêtes incidentes à $P^{\prime}$ soient plus proches de 1 que ne l'étaient celles des arêtes incidentes à $P$, 
2) la qualité de tous les tétraèdres de la boule de $P^{\prime}$ soit inférieure à celle du plus mauvais tétraèdre de la boule de $P$ :

$$
\forall K_{i} \in \mathcal{B}_{P^{\prime}} \quad Q_{K_{i}}<\max _{K_{j} \in \mathcal{B}_{P}}\left(Q_{K_{j}}\right)
$$

Cette méthode de bougé de points est appliquée sur tous les points du maillage après la procédure d'optimisation par retournements d'arêtes. Ces deux étapes sont réitérées tant que le maillage est modifié. Le critère d'amélioration stricte de la qualité des élements nous assure que l'algorithme convergera en un nombre fini d'itérations.

L'application du bougé de points permet de lisser les points du maillage c'està-dire de modifier la position des points pour que les arêtes du maillage aient une longueur la plus proche possible de 1 (dans la métrique prescrite). Par conséquent, la qualité moyenne des tétraèdres du maillage est améliorée.

\subsection{Méthodes de Delaunay anisotrope}

\subsubsection{Algorithme de construction isotrope}

Dans notre approche, l'insertion d'un point $P$ dans un maillage $\mathcal{T}_{i}$ est effectuée par un algorithme de type Bowyer-Watson (Bowyer, 1981, Watson, 1981). Cette méthode incrémentale basée sur le noyau de Delaunay s'écrit formellement comme suit :

$$
\mathcal{T}_{i+1}=\mathcal{T}_{i}-\mathcal{C}_{P}+\mathcal{B}_{P}
$$

avec $\mathcal{T}_{i+1}$ le nouveau maillage contenant le point $P, \mathcal{C}_{P}$ la cavité du point $P$ et $\mathcal{B}_{P}$ la boule du point $P$.

En d'autres termes, pour insérer le point $P$, il faut d'abord trouver tous les éléments qui composent sa cavité $\mathcal{C}_{P}$ pour les supprimer du maillage $\mathcal{T}_{i}$, puis étoiler le point $P$ sur les faces de la cavité (i.e., relier le point $P$ aux sommets constituant le bord de la cavité).

\subsubsection{Extension au cas anisotrope}

On s'intéresse maintenant à un problème de génération de maillage contrôlé. On considère un domaine représenté par une discrétisation initiale et on suppose données des spécifications de tailles et de directions (i.e., une métrique) en chaque point du maillage.

Dans le cas où la métrique spécifiée est de nature isotrope, c'est-à-dire lorsque seule la taille des éléments est définie, l'insertion d'un point peut se faire en suivant l'algorithme décrit à la section précédente.

Dans un contexte anisotrope, la procédure d'insertion doit être modifiée. En effet, une notion de longueur intervient dans la construction de la cavité, elle doit donc être adaptée pour respecter l'anisotropie prescrite. 
Dans le cadre classique, un élément $K$ fait partie de la cavité $\mathcal{C}_{P}$ s'il vérifie la relation suivante :

$$
\alpha(P, K)=\frac{d\left(P, O_{K}\right)}{r_{K}}<1
$$

où $d(.,$.$) représente la distance usuelle, O_{K}$ le centre de la sphère circonscrite au tétraèdre $K$ et $r_{K}$ le rayon de cette sphère.

Dans un contexte anisotrope, il faut transformer la formule précédente en remplaçant la distance usuelle par la distance définie par la métrique :

$$
\alpha_{\mathcal{M}}(P, K)=\frac{l_{\mathcal{M}}\left(P, O_{K}\right)}{r_{K}}<1
$$

Dans cette formule, $O_{K}$ est le point équidistant des sommets de $K$ au sens de la longueur de la métrique $\mathcal{M}$ et $r_{K}=l_{\mathcal{M}}\left(P_{1}, O_{K}\right)$ (si $P_{1}$ est un des sommets de $K$ ).

La relation [7] permet de définir la cavité dans un contexte anisotrope. Cependant, la métrique $\mathcal{M}$ variant d'un point à un autre, la détermination du centre $O_{K}$ et du rayon $r_{K}$ de la boule circonscrite au tétraèdre $K$ nécessite la résolution d'un système de trois équations qui est non linéaire ce qui n'est pas envisageable en pratique.

On cherche donc une solution approchée à notre problème. Pour cela, on commence par prendre en compte la métrique prescrite au point $P$, le critère [7] s'écrit donc :

$$
\alpha_{\mathcal{M}_{P}}(P, K)<1
$$

Le centre de la boule circonscrite au tétraèdre $K$ peut alors être calculé en résolvant le système suivant :

$$
\left\{\begin{array}{l}
l_{\mathcal{M}(P)}\left(O_{K}, P_{1}\right)=l_{\mathcal{M}(P)}\left(O_{K}, P_{2}\right) \\
l_{\mathcal{M}(P)}\left(O_{K}, P_{1}\right)=l_{\mathcal{M}(P)}\left(O_{K}, P_{3}\right) \\
l_{\mathcal{M}(P)}\left(O_{K}, P_{1}\right)=l_{\mathcal{M}(P)}\left(O_{K}, P_{4}\right)
\end{array}\right.
$$

avec $\left(P_{i}\right)_{i=1 . .4}$ les sommets du tétraèdre $K$.

La longueur d'un point $P_{i}$ à $O_{K}$ dans la métrique $\mathcal{M}(P)$ s'écrit de la manière suivante :

$m_{11}\left(x_{i}-O_{x}\right)^{2}+2 m_{12}\left(x_{i}-O_{x}\right)\left(y_{i}-O_{y}\right)+2 m_{13}\left(x_{i}-O_{x}\right)\left(z_{i}-O_{z}\right)$ $+m_{22}\left(y_{i}-O_{y}\right)^{2}+2 m_{23}\left(y_{i}-O_{y}\right)\left(z_{i}-O_{z}\right)+m_{33}\left(z_{i}-O_{z}\right)^{2}$

où $P_{i}=\left(\begin{array}{c}x_{i} \\ y_{i} \\ z_{i}\end{array}\right), O_{K}=\left(\begin{array}{c}O_{x} \\ O_{y} \\ O_{z}\end{array}\right)$ et $\mathcal{M}(P)=\left(\begin{array}{lll}m_{11} & m_{12} & m_{13} \\ m_{12} & m_{22} & m_{23} \\ m_{13} & m_{23} & m_{33}\end{array}\right)$ 
Calculer les coordonnées du centre $O_{K}$ revient donc à résoudre le système linéaire suivant :

$$
\left(\begin{array}{lll}
a_{2} & b_{2} & c_{2} \\
a_{3} & b_{3} & c_{3} \\
a_{4} & b_{4} & c_{4}
\end{array}\right)\left(\begin{array}{c}
O_{x} \\
O_{y} \\
O_{z}
\end{array}\right)=\left(\begin{array}{c}
d_{1} \\
d_{2} \\
d_{3}
\end{array}\right)
$$

avec

$$
\begin{aligned}
a_{i}= & 2\left(m_{11}\left(x_{i}-x_{1}\right)+m_{12}\left(y_{i}-y_{1}\right)+m_{13}\left(z_{i}-z_{1}\right)\right), \\
b_{i}= & 2\left(m_{22}\left(y_{i}-y_{1}\right)+m_{12}\left(x_{i}-x_{1}\right)+m_{23}\left(z_{i}-z_{1}\right)\right) \\
c_{i}= & 2\left(m_{33}\left(z_{i}-z_{1}\right)+m_{13}\left(x_{i}-x_{1}\right)+m_{23}\left(y_{i}-y_{1}\right)\right) \\
d_{i}= & m_{11} x_{i}^{2}+2 m_{12} x_{i} y_{i}+2 m_{13} x_{i} z_{i}+m_{22} y_{i}^{2}+2 m_{23} y_{i} z_{i}+m_{33} z_{i}^{2} \\
& -\left(m_{11} x_{1}^{2}+2 m_{12} x_{1} y_{1}+2 m_{13} x_{1} z_{1}+m_{22} y_{1}^{2}+2 m_{23} y_{1} z_{1}+m_{33} z_{1}^{2}\right)
\end{aligned}
$$

Cependant, la métrique prescrite au point $P$ est issue d'une interpolation, cette métrique ne rend donc pas forcément bien compte de l'anisotropie. Dans l'absolu, on souhaite prendre en compte les métriques aux quatre sommets de l'élément $K$ pour construire la cavité c'est-à-dire vérifier :

$$
\forall i=1, . ., 4 \quad \alpha_{\mathcal{M}\left(P_{i}\right)}(P, K)<1 .
$$

En pratique, on choisit un critère un peu moins restrictif. Le tétraèdre $K$ devra vérifier les deux relations suivantes pour appartenir à la cavité de $P$ :

$$
\left\{\begin{aligned}
\alpha_{\mathcal{M}_{P}}(P, K) & <1, \\
\sum_{i=1}^{4} \alpha_{\mathcal{M}\left(P_{i}\right)}(P, K)+\alpha_{\mathcal{M}_{P}}(P, K) & <5 .
\end{aligned}\right.
$$

Cependant, cette manière de construire la cavité d'un point n'assure pas l'étoilement du point $P$ sur les faces de celle-ci et les tétraèdres composant la cavité ne sont pas obligatoirement connexes. Il faut donc mettre en œuvre une procédure de correction de la cavité pour vérifier explicitement l'étoilement des tétraèdres de la cavité sur le point $P$. Pour cela, on teste si tous les nouveaux tétraèdres ont des volumes positifs. Lorsque ce n'est pas le cas, on corrige la cavité, ce qui signifie qu'on enlève des tétraèdres de celle-ci.

\subsection{Algorithme de remaillage}

L'algorithme de remaillage se décompose en deux parties : d'une part, l'optimisation des longueurs des arêtes au vu de la métrique prescrite et d'autre part, l'optimisation de la qualité des éléments.

Nous commençons donc par étudier les longueurs des arêtes : si une arête est trop longue, nous insérons un point sur celle-ci à l'aide de la procédure décrite à la 
section 3.5.2 ; à l'inverse, si elle est trop courte, nous la supprimons du maillage ( cf. 3.4.2). Ce procédé est itéré tant que des modifications sont faites. Pour assurer la convergence de cette partie de l'algorithme, lors de la suppression d'un point, un contrôle de la longueur des arêtes créées est effectué afin de s'assurer de ne pas générer des arêtes qui devraient être subdivisées par la suite.

Une fois cette phase terminée, la densité de points dans le maillage est conforme à celle prescrite par la métrique et nous cherchons à optimiser la qualité des éléments. Pour cela, nous appliquons au maillage des retournements d'arêtes (section 3.4.1) puis nous bougeons les points à l'aide de la procédure décrite précédemment (3.4.3). Ces deux phases d'optimisation de la qualité du maillage sont effectuées tant que des modifications sont possibles. Toutes ces opérations sur le maillage étant faites en augmentation stricte de la qualité des éléments, la convergence de l'algorithme est assurée.

\section{Etudes de flux d'air}

\subsection{Analyse du problème}

Pour simuler la propagation de l'air conditionné à l'intérieur d'une géométrie quelconque, il faut d'une part, savoir calculer l'écoulement d'air et d'autre part, pouvoir quantifier la répartition de température.

\subsubsection{L'écoulement d'air}

Soit $\Omega \in \mathbb{R}^{3}$ un domaine fermé borné. Il est bien établi que pour des températures comprises entre -20 et 100 degrés, l' air ambiant est un fluide newtonien compressible dont la densité est une fonction de la température. La vitesse $u$ et la pression $p$ sont donc gouvernées par les équations de Navier-Stokes pour les fluides visqueux compressibles avec une densité non constante $\rho$ (Landau et al., 1964), ce qui peut s'écrire comme suit (Glowinski, 1984, Pironneau, 1989) :

$$
\left\{\begin{aligned}
\rho\left(\frac{\partial u}{\partial t}+u \cdot \nabla u\right) \nabla \cdot S-\nabla p & =-\rho g e_{3}, \\
\frac{d \rho}{d t}+\nabla \cdot(\rho u) & =0 \quad \text { dans } \Omega,
\end{aligned}\right.
$$

où $S=\mu\left(\nabla u+\nabla u^{T}\right)$ est le tenseur de rigidité, $g$ la gravité, $\mu$ est la viscosité et $e_{3}$ le troisième vecteur de la base canonique.

Cependant, les écoulements traités étant à petit nombre de Mach et l'écart de température faible, nous pouvons appliquer l'approximation de Boussinesq aux équations de Navier-Stokes. Ceci signifie que $\theta$ varie peu autour d'une température moyenne $\theta_{0}$ et que ses effets sont uniquement pris en compte dans le terme $g \rho$.

Dans les équations [11], les variations de $\rho$ sont donc négligées à gauche et on divise par sa valeur moyenne $\rho_{0}$. La loi des gaz parfaits $p=\rho R \theta$ (dans laquelle $R$ désigne la constante des gaz parfaits divisée par la masse moléculaire du gaz) donne 
une loi inverse de la température $\theta$ à pression constante. Pour évaluer le terme de poussée $-e_{3} g \frac{\rho}{\rho_{0}}$, l'approximation de Boussinesq nous dit :

$$
\theta=\theta_{0}+\theta^{\prime}, \quad\left|\theta^{\prime}\right|<<\left|\theta_{0}\right| \Rightarrow \rho=\frac{p_{0}}{R\left(\theta_{0}+\theta^{\prime}\right)} \sim \frac{p_{0}}{R \theta_{0}}\left(1-\frac{\theta^{\prime}}{\theta_{0}}\right)
$$

Ce qui peut se réécrire de manière équivalente comme

$$
\frac{\rho-\rho_{0}}{\rho_{0}}=\frac{\theta-\theta_{0}}{\theta_{0}}
$$

Cependant, la variation de la densité est négligée partout sauf dans le terme de poussée qui est une fonction linéaire de la température $\theta$. Ce qui nous donne en définitive $\rho g e_{3} / \rho_{0} \sim e_{3} g \theta / \theta_{0}$ d'où le système :

$$
\left\{\begin{array}{rlr}
\frac{\partial u}{\partial t}+u \cdot \nabla u+\nabla p-\nu \Delta u & =-e_{3} g \frac{\theta}{\theta_{0}} & \\
\nabla \cdot u & =0 \quad \text { dans } \Omega,
\end{array}\right.
$$

où $p$ est la pression réduite (i.e., $\left.p / \rho_{0}\right)$.

Les frontières $\partial \Omega$ sont soumises à des conditions aux limites classiques (Pironneau, 1989, Medić et al., 1999) :

- vitesse spécifiée (condition aux limites de Dirichlet) :

$$
u=w \operatorname{sur} \Gamma_{1}
$$

- tractions spécifiées (condition aux limites de Neumann):

$$
-p+\left(\nu\left(\nabla u+\nabla u^{T}\right) . n . n\right)=0 \text { et }\left(\nu\left(\nabla u+\nabla u^{T}\right) . n . s\right)=0 \operatorname{sur} \Gamma_{2},
$$

où $\Gamma_{1} \cup \Gamma_{2}=\partial \Omega, n$ et $s$ représentent respectivement la normale unitaire sortante et la tangente unitaire correspondante.

La condition initiale est une vitesse donnée :

$$
u(x, 0)=u_{0}(x)
$$

\subsubsection{La répartition de la température}

La température est calculée en résolvant une équation d'advection-diffusion qui vient de l'équation de conservation de l'énergie dans laquelle on suppose que le flux est incompressible avec une densité constante et que les effets de la viscosité du fluide sont négligés : 


$$
\frac{\partial \theta}{\partial t}+u \nabla \theta-\kappa \Delta \theta=0 \text { dans } \Omega
$$

où $\kappa$ est la diffusion thermique.

Les frontières $\partial \Omega$ sont soumises aux conditions aux limites suivantes :

- température spécifiée (condition aux limites de Dirichlet) :

$$
\theta=\theta_{0} \operatorname{sur} \Gamma_{d}
$$

- condition aux limites de Fourier :

$$
\frac{\partial \theta}{\partial n}+a \theta+b\left(\theta^{4}-\theta_{e}^{4}\right)=0 \operatorname{sur} \Gamma_{f},
$$

où $\Gamma_{d} \cup \Gamma_{f}=\partial \Omega, \theta_{e}$ est la température extérieure et $a, b$ sont deux constantes réelles. $a$ représente le coefficient d'absorption et $b$ le coefficient de radiation.

\subsection{Discrétisation en temps}

On utilise un schéma aux différences finies semi-explicite de type Euler avec une correction sur la pression pour imposer l'incompressibilité. Soient $\delta t$ le pas de temps et $u^{n}$ l'approximation de $u$ au temps $n \delta t$. Notons $\tilde{u}^{n}$ la vitesse intermédiaire (qui ne vérifie pas l'hypothèse d'incompressibilité). On résout le système suivant :

$$
\left\{\begin{aligned}
\frac{\tilde{u}^{n}-u^{n-1}}{\delta t}+u^{n-1} \cdot \nabla u^{n-1}+\nabla p^{n-1}-\nu \Delta u^{n-1} & =e_{3} g \alpha \theta^{n-1} \\
\frac{\theta^{n}-\theta^{n-1}}{\delta t}+u^{n} \nabla \theta^{n}-\kappa \Delta \theta^{n} & =0
\end{aligned}\right.
$$

Pour retrouver l'incompressibilité de $\tilde{u}^{n}$, on utilise une projection sur le sousespace des champs à divergence nulle (voir (Chorin, 1967) ou (Rannacher, 1992)) et on résout pour $\phi$ :

$$
-\Delta \phi=-\nabla \cdot \tilde{u}^{n} \operatorname{sur} \Omega, \quad \frac{\partial \phi}{\partial n}=0 \operatorname{sur} \Gamma_{1}, \quad \phi=0 \operatorname{sur} \Gamma_{2}
$$

Puis on pose

$$
u^{n}=\tilde{u}^{n}-\nabla \phi, p^{n}=p^{n-1}-\frac{\phi}{\delta t} .
$$




\subsection{Discrétisation en espace et méthodes de résolution}

Soit $\Omega_{h}=\bigcup_{j} K_{j}$ une discrétisation du domaine de calcul $\Omega$ en tétraèdres.

Soient $V_{h}$ l'espace des fonctions affines continues sur la tétraédrisation et $V_{0 h}$ le sous-espace contenant la condition aux limites de Dirichlet. Soit $J_{0 h}=V_{0 h}^{3}$. La formulation de Petrov-Galerkin est : trouver $\tilde{u}_{h}-w_{h} \in J_{0 h}$ tel que $\forall v_{h} \in J_{0 h}$

$$
\begin{aligned}
& \int_{\Omega_{h}} \frac{\tilde{u}_{h}^{n}-u_{h}^{n-1}}{\delta t} v_{h}+\int_{\Omega_{h}} u_{h}^{n-1} \nabla u_{h}^{n-1} v_{h}+\int_{\Omega_{h}} \nu \nabla u_{h}^{n-1} \nabla v_{h} \\
& +\int_{\Omega_{h}} \nabla p^{n-1} \cdot v_{h}+\int_{\Omega_{h}}\left(\nabla p^{n-1}+u_{h}^{n-1} \nabla u_{h}^{n-1}\right) \cdot G_{1}^{K}\left(u_{h}^{n-1}, v_{h}\right)=\int_{\Omega_{h}} f v_{h}
\end{aligned}
$$

où $w_{h}$ est une extension dans $\left(V_{h}\right)^{3}$ des conditions aux limites et $G_{1}^{K}$ sont les fonctions de stabilisation données par le schéma PSI (Deconink et al., 1993); notons que la dérivée en temps et le terme visqueux sont supprimés du terme de stabilisation. Pour résoudre ces équations, nous utilisons une méthode de mass lumping pour le terme en temps.

La discrétisation de [21] est faite classiquement sur $V_{0 h}$ et ce problème de Poisson est résolu en utilisant une méthode de gradient conjugué avec un préconditionneur diagonal.

Pour la température, on cherche un $\theta_{h}^{n}-\theta_{0 h} \in \Theta_{0 h}$ avec $\forall \tau_{h} \in \Theta_{0 h}=\left\{\tau_{h} \in\right.$ $\left.V_{h},\left.\tau_{h}\right|_{\Gamma_{1}}=0\right\}$ :

$$
\begin{aligned}
& \int_{\Omega_{h}} \frac{\theta_{h}^{n}-\theta_{h}^{n-1}}{\delta t} \tau_{h}+\int_{\Omega_{h}} \kappa \nabla \theta_{h}^{n-1} \nabla \tau_{h}+\int_{\Omega_{h}} u_{h}^{n} \nabla \theta_{h}^{n-1} \tau_{h} \\
& +\int_{\Gamma_{2 h}} \kappa\left(a \theta_{h}^{n}+b\left(\theta_{h}^{n}-\theta_{e}\right)\left(\left(\theta_{h}^{n-1}\right)^{2}+\theta_{e}^{2}\right)\left(\theta_{h}^{n-1}+\theta_{e}\right)\right) \tau_{h}=0
\end{aligned}
$$

Le système non symétrique obtenu est résolu en utilisant une technique itérative de GMRES (Generalized Minimal Residual). Dans notre cas, nous nous servons d'une version spécifique du GMRES qui permet l'implémentation d'un préconditionneur variable avec un protocole de communication inverse (Medić et al., 2001).

\subsection{Apport du parallélisme}

\subsubsection{Motivation}

Dans le cadre de simulation d'air conditionné, nous sommes amenés à travailler avec des géométries complexes et donc avec des maillages contenant un nombre important d'éléments. Certes ces simulations peuvent tourner sur une station de travail 
(par exemple un PC à $2 \mathrm{Ghz}$ ) mais elles demandent un temps conséquent, temps qui est d'autant plus grand que les phénomènes calculés se produisent sur des durées de l'ordre de dizaines de secondes. Par exemple, pour réaliser une simulation sur un maillage d'environ 1300000 éléments, il faut compter un peu plus de 4 heures pour effectuer 100 pas de temps, un pas de temps correspondant en pratique à une microseconde.

Les techniques d'adaptation de maillages mises en œuvre permettent de réduire significativement le nombre de nœuds des maillages tout en simulant avec précision les phénomènes physiques. En effet, ces techniques génèrent des maillages dans lesquels la densité des éléments traduit les variations de la solution. Cependant, les géométries dans lesquelles sont calculées les problèmes d'air conditionné sont souvent assez complexes et comportent un certain nombre de détails (tels que des meubles dans une maison ou le siège d'une voiture). Ces détails impliquent un raffinement local du maillage pour respecter la géométrie. Les techniques d'adaptation de maillage n'ont donc pas d'impact sur ces parties de la géométrie et les maillages obtenus suite à l'adaptation de maillage contiennent encore un nombre conséquent de mailles. Dans l'optique d'accélérer encore les temps de calcul de nos simulations, nous nous sommes donc tournés vers le parallélisme.

La boucle d'adaptation de maillage se compose de différents éléments, indépendants les uns des autres dont les deux principaux sont les mailleurs et les codes de calcul. Dans une telle boucle, le temps dévolu au maillage (c'est-à-dire le temps nécéssaire à la génération du maillage adapté) est inférieur à 5 minutes pour un maillage avoisinant le million d'éléments. Par contre, comme nous l'avons déjà dit, le temps de calcul dû aux solveurs se compte en heures. Il nous a donc paru essentiel de paralléliser les solveurs pour obtenir des temps de calcul raisonnables.

\subsubsection{Principe et outils de la parallélisation}

Pour effectuer les calculs en parallèle, on utilise une technique de décomposition de domaine pour partitionner le domaine initial en sous-domaines. Dans notre cas, cette décomposition est faite par rapport aux tétraèdres du maillage (ce qui signifie que certains nœuds du maillage sont communs à plusieurs sous-domaines). Pour que le calcul soit rapide, la décomposition du maillage doit répondre du mieux possible à deux critères : la minimisation des communications entre les sous-domaines et la répartition équitable de la taille des sous-domaines (ici au sens du nombre de nœuds). Pour décomposer le maillage, nous avons utilisé la bibliothèque Metis (Karypis et al., 1998). Met is est une famille de programmes permettant de partitionner des graphes ou des maillages de type éléments finis non structurés tout en réduisant le plus possible les interfaces entre les différentes partitions, sous réserve d'équilibre. L'algorithme principal utilisé par Metis est basé sur un schéma de partitionnement de graphes multi-échelles décrit dans (Karypis et al., 1998).

Dans le cas de codes éléments finis, pour calculer la solution approchée en un point du maillage, seuls les points « voisins » sont nécessaires, autrement dit les points ayant une arête commune. Pour les points « intérieurs » à un sous-domaine, (i.e., pour les 
points n'appartenant qu'à un seul sous-domaine), le calcul s'effectue donc de la même façon que dans le cas d'un calcul séquentiel. Seuls les points sur une interface, c'est-àdire les points appartenant à au moins deux sous-domaines, ne disposent pas de toutes les informations nécessaires pour faire les calculs. Ils ont donc besoin des valeurs que possèdent un ou plusieurs sous-domaines voisins. Il y a alors communications point-àpoint entre un ou plusieurs sous-domaines bien identifiés. De plus, à chaque itération en temps, certaines quantités nécessitent un calcul qui fait intervenir l'ensemble du domaine : par exemple pour évaluer le pas de temps. Cela se fait à l'aide d'une communication collective (tous les sous-domaines envoient à un seul leur valeur locale) et le destinataire détermine la valeur acceptable (opération de réduction) puis la diffuse à tous les sous-domaines (opération de diffusion). Les communications sont gérées grâce à la bibliothèque MPI ${ }^{1}$.

\subsubsection{Performancelévaluation}

Pour évaluer les performances des codes qui ont été parallélisés, nous avons calculé l'indice d'efficacité (Havé, 2004).

Définition 7 L'indice d'efficacité est défini comme le complément de la perte relative de puissance de calcul induit par l'accroissement du nombre de processeurs. L'indice d'efficacité $I_{n}$ correspondant au calcul sur $n$ processeurs est calculé par la formule suivante :

$$
I_{n}=\frac{\text { temps de calcul sur } 1 \text { processeur }}{\text { temps de calcul sur } n \text { processeurs } * n} .
$$

Cet indice permet de mesurer le taux d'utilisation de la puissance des machines. Un calcul parallèle est composé de deux parties : le calcul proprement dit qui est le même quel que soit le nombre de processeurs et le temps de synchronisation c'est-à-dire le temps passé dans les communications entre les différents processeurs. Idéalement, on voudrait que $100 \%$ de la puissance des ordinateurs soit utilisée pour les calculs, l'indice d'efficacité serait alors égal à 1 (c'est le cas lors d'un calcul séquentiel).

Dans le but de quantifier le temps gagné grâce à la parallélisation, nous avons effectué des calculs correspondant à 10 pas de temps. Nous avons utilisé d'une part, un maillage comportant 65399 nœuds et 320826 éléments (cas 2) et d'autre part, un maillage plus conséquent composé de 248933 points et 1333334 tetraèdres (cas 1). Les calculs ont été effectués sur une machine de type $M I M D$ : le cluster Hydre du Laboratoire J.-L. Lions qui fonctionne sous Debian/GNU-Linux. Ce cluster est composé de deux types de machines, que l'on nommera HydreI et HydreII. HydreI est un ensemble de $8 \mathrm{PC}$ de type Bi-Pentium4 Xeon à 2, $4 \mathrm{Ghz}$ avec 2 Go de RAM connectés à 1 Gigabit et HydreII est composé de 8 machines de type Pentium3 à 1, 266 Ghz avec 1 Go de RAM connectées à 100 Megabit.

Les indices d'efficacité calculés pour les différents cas tests sont représentés sur la figure 2. Dans le cas 1 du calcul de flux d'air, on observe que l'indice d'efficacité

1. Message Passing Interface. 
augmente très fortement pour 2 processeurs. On en déduit que la taille de maillages sur chaque processeurs tient dans la mémoire cache et donc que l'accès mémoire est beaucoup plus rapide. L'indice décrôit ensuite doucement, traduisant ainsi l'augmentation du nombre de communications. On observe le même type de phénomènes pour tous les autres cas du calcul de flux d'air.

Concernant l'autre solveur, pour le cas 1 , l'indice d'efficacité est maximum pour 3 ou 6 processeurs (selon le type de machine), on retrouve donc encore une fois l'accroissement de l'indice dû à l'utilisation de la mémoire cache. Par contre pour le cas 2 (plus petit en nombre de points), l'indice décroît beaucoup plus rapidement.
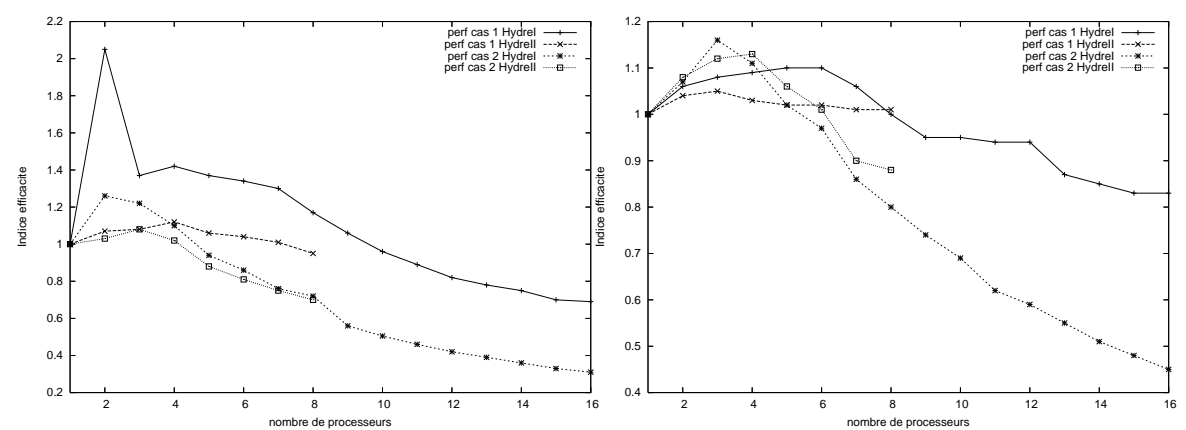

Figure 2. Performance en fonction du nombre de processeurs : à gauche, pour le solveur des équations de Navier-Stokes et à droite, pour le solveur de thermique

\section{Exemple d'application}

On s'intéresse dans cette section au réchauffement d'une maison. Cette maison compte trois étages et est partiellement meublée. La maison mesure environ $10 \mathrm{~m} \times$ $10 \mathrm{~m} \times 10 \mathrm{~m}$. L'intérêt de cette étude est de montrer la faisabilité d'un calcul d'air conditionné dans une géométrie complexe comportant un certains nombre de détails (tables, chaises...).

\subsection{Calcul de l'écoulement}

Pour la simulation, on impose trois entrées d'air chaud sur un mur de la maison (une entrée par étage). Une condition de Dirichlet est donc prescrite sur ces portions de la frontière. Dix sorties d'air sont prescrites un peu partout dans la maison pour permettre à l'écoulement de s'installer. On impose sur les murs et les meubles des conditions d'absorption ou de radiation. La vitesse d'entrée de l'air est $2 \mathrm{~m} . \mathrm{s}^{-1}$. Le calcul a été effectué dans une boucle d'adaptation de maillage dont les différents paramètres sont donnés à la section suivante. Des lignes de courant de l'écoulement sont 
représentées sur la figure 3 et des isosurfaces à différents temps sont montrées sur la figure 4.

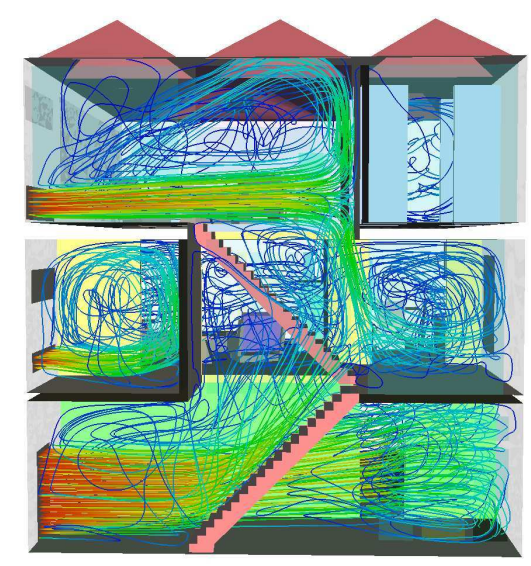

Figure 3. Ligne de courant de l'écoulement à $t=26$ secondes
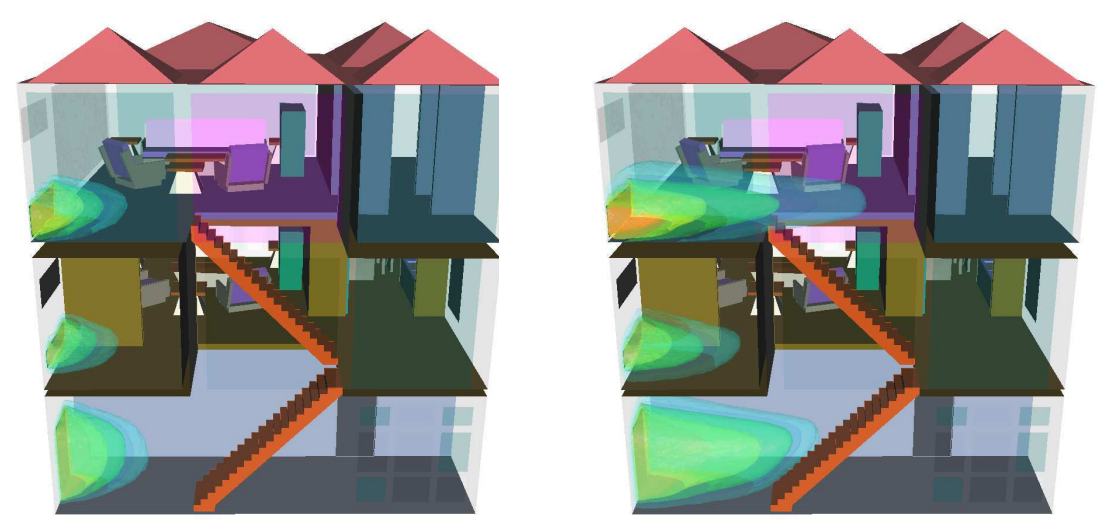

Figure 4. Isosurfaces de température à $t=2$ et 26 secondes

\subsection{Adaptation de maillages}

Pour cette simulation, le maillage a été adapté à chaque fois que 2 secondes de temps physique étaient écoulées. La construction de la métrique est basée sur les trois composantes de la vitesse. On impose une erreur de 0,015 , une taille de maillage minimale (resp. maximale) de 0,15 mètre (resp. 1,7 mètres). On considère un maillage 
initial isotrope uniforme comportant 1915548 éléments (figure 5). Le module de remaillage par modifications locales est utilisé pour adapter le maillage. Pour effectuer deux secondes de temps physique, il a fallu, à l'itération 13 environ 15 heures.

On observe sur la figure 5 le maillage adapté créé à l'itération 13. Comme prévu, dans les zones où l'air s'écoule, le maillage a été raffiné, en particulier au niveau des frontières de celui-ci. De plus, dans les pièces où l'air ne circule pas, les tétraèdres créés sont de tailles maximales. La figure 6 permet de voir plus précisément l'anisotropie du maillage dans une zone où l'air s'écoule.

Le tableau 1 donne un récapitulatif des caractéristiques des maillages aux itérations 1,5 et 13 .

A toutes les itérations, les maillages construits correspondent à la carte de métrique prescrite : les indices d'efficacité sont toujours supérieurs à 0,82 . De plus, la qualité des tétraèdres du maillage est globalement bonne : plus de $92 \%$ de tétraèdres ont une qualité inférieure à 3 .

\begin{tabular}{|c||c|c|c|c|c|}
\hline & $n p$ & $n e$ & CPU (en sec.) & $\tau$ & $Q<3($ en \%) \\
\hline initial & 351441 & 1915548 & & & \\
\hline 1e adaptation & 152621 & 698051 & 655 & 0,8277 & 92,13 \\
\hline 5e adaptation & 176786 & 838363 & 561 & 0,8313 & 95,28 \\
\hline 13e adaptation & 202741 & 990700 & 661 & 0,8438 & 96,92 \\
\hline
\end{tabular}

Tableau 1. Caractéristiques des maillages pour la simulation dans la maison

\section{Conclusion}

Nous avons présenté dans cet article un travail sur l'adaptation de maillage anisotrope $3 \mathrm{~d}$ pour les problèmes d'aérothermique dans les bâtiments. Pour simuler ces phénomènes en un temps raisonnable, nous avons fait le choix de l'adaptation de maillage anisotrope pour réduire le nombre de nœuds du maillage tout en contrôlant l'erreur d'approximation. Les résultats présentés démontrent la faisabilité et l'efficacité de l'approche globale.

Cependant, en raison du bruit numérique sur la solution calculée, les rapports d'anisotropie prescrits par la métrique sont assez faibles. Pour palier ce problème, il faudrait filtrer ce bruit, par exemple en utilisant une convolution avec une fonction régularisante (Hecht, 2005). De plus, un traitement de lissage de métrique serait aussi nécessaire pour gérer les sauts de métrique (Borouchaki et al., 1998). Enfin, pour être tout à fait réalistes, il faudrait traiter le cas de fluides turbulents en rajoutant un modèle de turbulence aux équations de Navier-Stokes. 

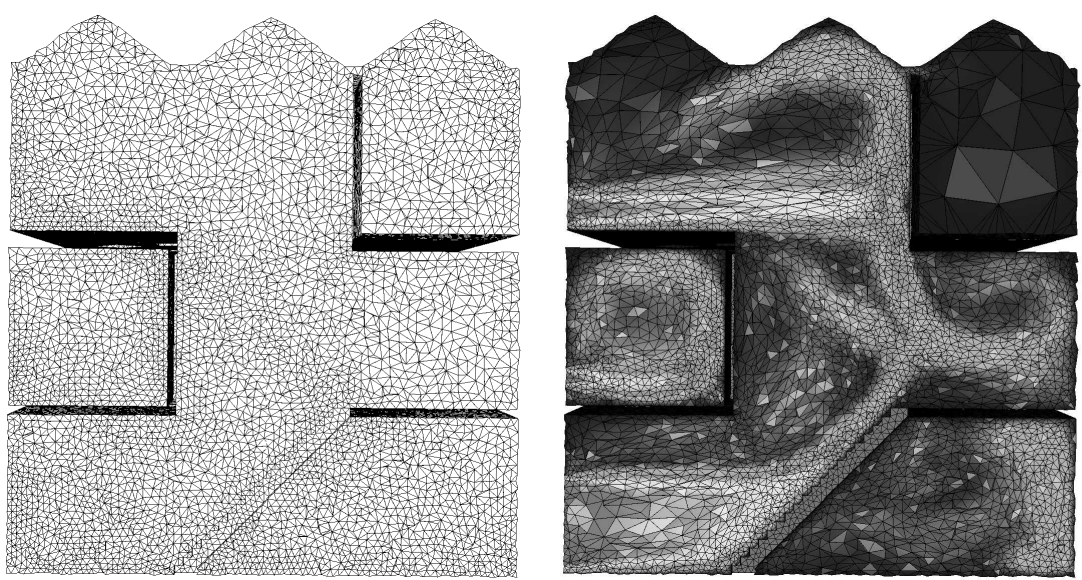

Figure 5. Coupe volumiques dans le maillage initial du domaine et dans le maillage adapté à $t=26$ secondes

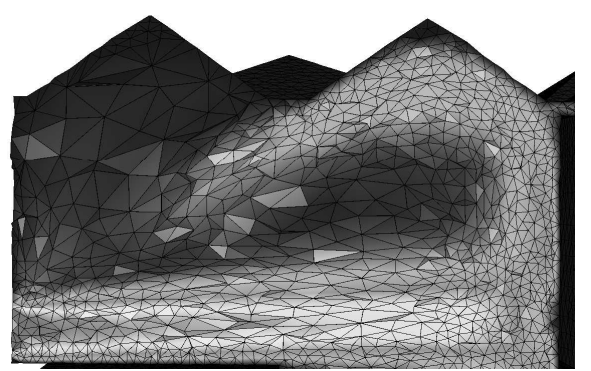

Figure 6. Agrandissement de coupes volumiques dans le maillage adapté à $t=26$ secondes

\section{Bibliographie}

Alauzet F., Adaptation de maillage anisotrope en trois dimensions. Application aux simulations instationnaires en Mécanique des Fluides, Thèse de doctorat, Université de Montpellier II, 2003.

Alauzet F., Frey P., Estimateur d'erreur géométrique et métriques anisotropes pour l'adaptation de maillage. Partie 1 : aspects théoriques, Rapport de recherche $n^{\circ}$ RR-4759, INRIA, 2003.

Borouchaki H., Hecht F., Frey P., « Mesh gradation control », Int. J. Num. Meth. Engrg, vol. 43, n 6, p. 1143-1165, 1998.

Bowyer A., «Computing Dirichlet tesselations », The Comp. J., vol. 24, n² 2, p. 162-167, 1981. 
Chorin J., « A numerical method for solving incompressible viscous flow problems », J. Compt. Phys., vol. 2, p. 12-26, 1967.

Ciarlet P., Error Estimates for Elliptic Problems, in Handbook of Numerical Analysis, vol. II of Finite Element methods (Part 1), P.G. Ciarlet and J.L. Lions Eds, North Holland, 1991.

Coupez T., « Génération de maillage et adaptation de maillage par optimisation locale », Revue européenne des éléments finis, vol. 9, $\mathrm{n}^{\circ}$ 4, p. 403-423, 2000.

Deconink H., Struijs R., Bourgois G., Roe P., « Compact advection schemes on unstructured grids », VKI Lecture Series, 1993.

Fortin M., «Etude numérique d'estimations d'erreur a posteriori », Revue européenne des éléments finis, vol. 9, No4, p. 467-486, 2000.

Frey P., George P.-L., Maillages : applications aux éléments finis, Hermès Science, Paris, 1999.

George P.-L., Borouchaki H., Frey P., Laug P., Saltel E., Mesh generation and mesh adaptivity : theory, tecniques, Encyclopedia of computational mechanics, E. Stein, R. de Borst and T.J.R. Hugues ed., John Wiley \& Sons Ltd., 2004.

Girault V., Raviart P.-A., Finite element methods for Navier-Stokes equations : theory and algorithms, Springer-Verlag, New-York, 1986.

Glowinski R., Numerical methods for nonlinear variational problems, Springer-Verlag, NewYork, 1984.

Havé P., Méthodes Multipôles Rapides pour l'électromagnétisme : Parallélisme et Basses Fréquences, Thèse de doctorat, Université Pierre et Marie Curie - Paris 6, 2004.

Hecht F., «A few snags in mesh adaptation loops », Proc. of 14th Int. Meshing Roundtable, San Diego, CA, USA, 2005.

Karypis G., Kumar V., «A fast and high quality multilevel scheme for partitioning irregular graphs », SIAM J. Sci. Comput., vol. 20, n 1, p. 359-392 (electronic), 1998.

Landau L., Lipschitz F., Mécanique des fluides, Mir, 1964.

Leservoisier D., George P.-L., Dervieux A., Métrique continue et optimisation de maillage, Rapport de recherche $n^{\circ}$ RR-4172, INRIA, 2001.

Medić G., Mohammadi B., NSIKE : An Incompressible Navier-Stokes Solver for Unstructured Meshes, Rapport technique $\mathrm{n}^{\circ}$ RT-3644, INRIA, 1999.

Medić G., Mohammadi B., «Injection/Suction Boundary Conditions for Fluid/Structure Interaction Simulation in Incompressible Flows », Int. J. Numer. Meth. Fluids, vol. 40, p. 275290, 2001.

Mohammadi B., Pironneau O., Analysis of the K-Epsilon Turbulence Model, Wiley, 1994.

Pain C., Humpleby A., de Oliveira C., Goddard A., « Tetrahedral mesh optimisation and adaptivity for steady-state and transient finite element calculations », Comput. Methods Appl. Mech. Engrg, vol. 190, p. 3771-3796, 2001.

Pironneau O., The finite element method for fluids, Wiley, Chicester, 1989.

Rannacher R., « On Chorin's projectino method for the incompressible Navier-Stokes equations », Lecture Notes in Mathematics 1530,p. 167-183, 1992.

Remacle J.-F., Li X., Chevaugeon N., Shepard M., « Transient mesh adaptation using conforming and non conforming mesh modifications », Proc. of 11th Int. Meshing Roundtable, Ithaca, NY, USA, 2002. 
Tam A., Ait-Ali-Yahia D., Robichaud M., Moore M., Kozel V., Hasbashi W., « Anisotropic mesh adaptation for 3D flows on structured and unstructured grids », Comput. Methods Appl. Mech. Engrg, vol. 189, p. 1205-1230, 2000.

Vallet M., Génération de maillages anisotropes et adaptatifs, Thèse de doctorat, Université de l’Université Paris VI, 1992.

Watson D., « Computing the n-dimensional Delaunay Tesselation with applications to Voronoï polytopes », Computer Journal, vol. 24, n² 2, p. 167-172, 1981. 
